\title{
La formation à la mixité scolaire à la mesure du genre
}

Dominique Gauthiez-Rieucau

\section{(2) OpenEdition}

1 Journals

Édition électronique

URL : http://journals.openedition.org/trema/1080

DOI : 10.4000/trema.1080

ISSN : 2107-0997

Éditeur

Faculté d'Éducation de l'université de Montpellier

Édition imprimée

Date de publication : 1 juin 2010

Pagination : 23 - 33

ISSN : 1167-315X

\section{Référence électronique}

Dominique Gauthiez-Rieucau, «La formation à la mixité scolaire à la mesure du genre », Tréma [En ligne], 32 | 2010, mis en ligne le 01 juin 2012, consulté le 02 mai 2019. URL : http:// journals.openedition.org/trema/1080 ; DOI : 10.4000/trema.1080

Ce document a été généré automatiquement le 2 mai 2019.

Trema 


\title{
La formation à la mixité scolaire à la mesure du genre
}

\author{
Dominique Gauthiez-Rieucau
}

La quatrième Convention interministérielle pour l'égalité entre les filles et les garçons, les femmes et les hommes dans le système éducatif (2006-2011) invite les acteurs et actrices "à écarter tout stéréotype lié au sexe, à faire évoluer les représentations, à diversifier l'orientation des filles et des garçons, à valoriser la place et le rôle des femmes, à diffuser les études sur le genre » 1 .

Il s'agit désormais d'introduire la formation à la mixité scolaire dans le processus de masterisation et de professionnalisation.

Car l'enjeu de la formation est éducatif, pédagogique et sociétal dans un contexte d'inclusion normative (Margaret MEAD, 1963 ; Elena GIANINI BELOTTI, 1973) et de valence différentielle (Françoise HERITIER, 1996) où pas plus la mixité - installée de manière pragmatique et généralisée tardivement en 1975 - que la perspective réaliste d'une société égalitaire, n'ont été anticipées: il implique tous les niveaux de l'institution Éducation nationale, de la maternelle à l'enseignement supérieur. En fait, le ministère de l'Éducation nationale et de la recherche n'en disconvient pas et même l'exprime explicitement : 
«L'extraordinaire, dans le combat pour l'égalité entre les sexes, c'est qu'il est toujours à recommencer et que l'actualité en donne sans cesse un éclairage neuf. Or ce combat a derrière lui l'histoire de l'humanité - ou peu s'en faut. Non que nos sociétés n'aient, depuis quelque cinquante ans, considérablement évolué. Mais tout démontre aujourd'hui encore que, dans les faits, la femme n'est jamais tout à fait l'égale de l'homme ». (BOEN, 2000, Préambule).

Les résistances à la généralisation des formations à la mixité - formations pourtant nécessaires pour contrer les inégalités de genre - dans les IUFM devenus des écoles annexes des universités en formation initiale supérieure, comme à tous les niveaux de l'institution Éducation nationale en formation continue, semblent avoir des origines communes: certains obstacles sont liés à l'inconscient collectif, d'autres relèvent de l'absence d'une volonté politique soutenue.

\section{La difficile fondation de la mixité}

\section{Les paradoxes du système éducatif : un discours républicain explicite égalitaire mais une réalité implicite de terrain inégalitaire}

Rien de vraiment surprenant à la médiatisation du débat qui déborde largement les sciences de l'éducation autour de la mixité , face à l'acuité de cette question centrale, posée au cœur de l'Institution française : la mixité, généralisée par le ministre René HABY ${ }^{3}$ se voit questionnée dans les médias qui sollicitent les témoignages de chercheurs et d'universitaires. À l'origine de cette remise en question se trouve un double constat: d'une part les difficultés relationnelles entre filles et garçons, incluant le déficit d'éducation au respect mutuel de l'autre sexe à l'égard des professeur(e)s, incivilités croissantes, pratiques langagières et violences sexistes voire sexuelles qui ne concernent pas seulement les banlieues ou quartiers sensibles mais sont une donne sociétale évaluée par la mise en place du logiciel Signa dans les établissements secondaires ${ }^{4}$; d'autre part, le constat paradoxal des meilleurs résultats des filles qui, en outre, redoublent moins, obtiennent plus de mentions, cela des premiers apprentissages jusqu'au Baccalauréat +2 mais ne s'orientent pas pour autant vers les filières d'excellence, en particulier scientifiques (MARRY, 2004, pp. 46-63) et ne diversifient pas non plus leurs projets professionnels, victimes de mécanismes d'autocensure ou de stratégies raisonnables d'adaptabilité aux résistances sociétales, paradoxe que Michèle FERRAND (2004, p. 61) qualifie de « victoire à la Pyrrhus ».

Il peut être opportun de rappeler à ce point de la réflexion que l'histoire de la scolarisation des filles (LELIEVRE, 1991) a été lente, inversée par rapport à celle des garçons puisqu'elle part de la base et non de la volonté de former les élites du pays, décalée avec presque un demi-siècle de retard dans le primaire et quasiment un siècle dans l'enseignement secondaire: véritable "révolution silencieuse », pour reprendre la formule attribuée à Antoine PROST et sûrement aussi "révolution respectueuse " (MARRY, 2004). Si le décret Bérard aligne tardivement, en 1924, les programmes des filles et des garçons en vue de l'examen du baccalauréat, c'est que les études n'étaient nullement destinées à autoriser l'accès de la sphère publique aux filles mais à en faire des épouses et des mères moins ignorantes. Et la majorité des "grandes écoles » supérieures ne leur seront ouvertes que dans la foulée des années soixante-dix. Cependant le 
rattrapage des garçons par les filles a lieu dès 1972, date à laquelle le nombre des bachelières dépasse celui des bacheliers.

\section{La mixité, une idée neuve servie par les politiques d'égalité}

L'hypothèse, formulée par Michelle ZANCARINI-FOURNEL (THEBAUD et ZANCARINI, 2003, pp. 11-19), est que la mixité n'a jamais été pensée de fait, mais qu'elle s'est installée de manière pragmatique. Si plus de la moitié des communes dites rurales, en 1933, ont une école où est instaurée une « co-instruction » de fait, elles le doivent à un souci d'économie budgétaire : c'est le résultat de la "gémination", le jumelage de classes à petits effectifs qui passe par le regroupement par âges de filles et de garçons. La question n'est pas posée en terme de rapports sociaux de sexe : cette co-instruction de fait ne recoupe pas les idées exprimées lors du Congrès de Lille en 1905 par les Amicales des institutrices et instituteurs, à savoir une coéducation pensée dans une dynamique sociétale égalitaire (ibid., p. 14) ; le concept « d'éducation intégrale » théorisé par Paul ROBIN et expérimenté dans un orphelinat de l'Oise de 1880 à $1894^{5}$ (ibid., p. 312) relevait d'une idéologie parfaitement novatrice pour l'époque. En est signifiant le fait qu'il consacrera la fin de sa vie à la cause de « la libre maternité ».

Michelle ZANCARINI-FOURNEL précise que le mot «mixité » apparaît pour la première fois dans une circulaire du 3 juillet 1957, où voisinent les trois termes "gémination, coéducation, mixité»; la réforme BERTHOIN de 1959 légalise les lycées mixtes nouvellement construits et il en va de même pour les CES lors de la réforme FOUCHET en 1963. Elle insiste sur le fait qu'en 1965, les «administrateurs de l'Éducation nationale » eux-mêmes présentent la coéducation «comme une conséquence de l'évolution de la société ${ }^{\star}$. En 1976, les décrets d'application de la loi HABY généralisent l'organisation des «classes mixtes » à tous les degrés de l'enseignement mais "le terme même de mixité n'apparaît pas dans ces textes qui autorisent la pratique conjointe des deux sexes dans l'enseignement (Ibid., p. 15)».

L'institutionnalisation récente du genre (GAUTHIEZ-RIEUCAU, 2007) révèle a contrario cette absence d'anticipation éducative et pédagogique: en appui sur les Conventions interministérielles Égalité entre filles et garçons, femmes et hommes, Dominique TORSAT éclaire comment les politiques d'égalité à l'école viennent combler ce vide (TORSAT, 1999). Le colloque du Conseil national des programmes tenu au CNAM le 6 avril 2004 «L'enseignement est-il mixte? » ou le colloque national tenu à Paris le 27 mai 2004 « De la mixité... à l'égalité dans le système éducatif» témoignent des avancées de la conscientisation du genre. Il apparait enfin dans le discours explicite et dans la loi que la mixité ne suffit pas à instaurer l'égalité.

\section{La socialisation différenciée, à l'avantage des garçons}

Soit un discours républicain explicite censé viser une société égalitaire... mais une réalité de terrain implicite inégalitaire fondée sur la socialisation différenciée dès la maternelle et l'école primaire, comme l'expose Claude ZAIDMAN $(1992,1996)$ et au fil des études secondaires, comme le confirme Nicole MOSCONI (1989). Soit une école qui régénère inlassablement et transmet les stéréotypes dans tous les aspects de l'éduquer - enseigner - orienter et qui biaise obligatoirement l'enjeu sociétal proclamé. 


\section{D'une logique binaire d'affrontement à un projet démocratique}

Il est vrai que le devenir de l'école est imbriqué dans un projet sociétal démocratique qui considère citoyen et citoyenne dans un égal respect. Ce projet historique plonge ses racines profondes dans le passé, fondement d'une République nouvelle, celle qu'initiaient POULAIN DE LA BARRE au 17e siècle, Marie-Olympe DE GOUGES ${ }^{7}$ et CONDORCET au 18e siècle, les fondateurs de l'école laïque au 19e siècle et Camille SEE, à l'origine des lycées de jeunes filles voire des plumes éclairées, telle celle de l'écrivain Victor MARGUERITTE qui écrivit son ouvrage au titre précurseur, Ton corps est à toi, en 1927. Une République, qui, après avoir avoué le déni historique de la moitié féminine de la société, derrière le grossier subterfuge du suffrage dit « universel » dès 1848 et jusqu'en 1944, accepte de penser, d'anticiper les conséquences sociétales majeures de la scolarisation des filles à travers tout le cursus. De penser le devenir des individus, femmes ou hommes indépendamment de leur sexe, cela dans la sphère publique, le monde du travail, la politique, l'art comme dans la sphère privée et la famille. De penser les masculinités plurielles (CASTELAIN-MEUNIER, 1988) et les nouvelles parentalités (THERY, 1993, 2001) dans une société de " controverse démocratique » qui vise l'égalité - altérité, à l'instar de Geneviève FRAISSE, historienne ex-députée de l'Union européenne. Donc de conscientiser les inégalités de genre : à l'inverse du refoulement dans les normes imposées, d'où le rôle incontournable des acteurs professionnels de l'école laïque.

\section{Mixité inaccomplie et nécessaire conscientisation du genre par les enseignants}

\section{Un nouveau concept au service de l'égalité filles - garçons, femmes - hommes}

Le genre présente l'avantage d'intégrer le postulat ontologique de l'égale dignité de la personne humaine, quel que soit son sexe et la légitime aspiration à une égalité en droits et en situations ${ }^{8}$. Le genre est un concept transdisciplinaire. Pour faire simple, le genre est le « sexe social » résultat d'une construction culturelle. Gender en anglo-américain genere en italien - est le terme spécifique utilisé en sciences humaines pour permettre de conscientiser les rôles de sexe. Dans sa complète signification le concept de genre indique la dimension sociétale plénière du sexe, bio - construite historiquement au gré des étapes scientifiques et techniques (par ex. l'avant et l'après contraception orale), dans une société donnée, incluant tous les aspects culturels: croyances, représentations, mentalités, ressentis et comportements9. Car la construction des identités sexuées des garçons et des filles, les rôles attribués aux femmes et aux hommes à travers les différentes civilisations varient dans l'espace et le temps.

Le terme polysémique s'inscrit en premier lieu dans le domaine grammatical pour qualifier un substantif dit de genre masculin ou féminin, où il exprime une dualité, évoque un monde binaire catégorisé, hiérarchisé, autrement dit en absolue contradiction avec le sens du terme genre, tel que traduit de l'américain gender - terme apparu dans la controverse des années cinquante autour du jeune Bruce - Brenda - David suivi par MONEY, 1955 ; utilisé au plan de la recherche par le psychiatre américain Robert STOLLER in Sex and gender, 1968 puis par la sociologue britannique Ann OAKLEY, in Sex Gender and 
Society, 1972 -: le genre doit être entendu comme un concept ontologique de développement de la personne indépendamment de son sexe biologique et un instrument heuristique transdisciplinaire. À l'opposé d'une vision binaire, il remet donc la sexuation de l'individu « à sa juste place » et réhabilite l'élève dans ses potentialités spécifiques.

Ce sont les années 1980 qui opèrent la jonction transdisciplinaire entre les recherches sur le genre et les études sociales et culturelles sur la science. Le concept pénètre progressivement l'histoire et la sociologie. L'ouvrage devenu classique de Joan SCOTT (1986) est traduit en français en 1988 par Michèle RIOT-SARCEY. Le genre devient un outil de dé - reconstruction dans la mouvance postmoderne (Butler, 1990) ; il est décliné dans le domaine sociologique (MATHIEU, 1991) et amené à dénoncer la domination masculine (HURTIG, KAIL et ROUCH, 1991) ; puis transféré dans une démarche économico - sociale de type marxisant (DELPHY, 1998). Enfin la conceptualisation aboutie permet d'historiciser les études genrées et de les articuler avec d'autres relations de pouvoir en confrontant la problématique du genre à d'autres variables, l'ethnie, l'identité régionale ou nationale, la classe, la caste (MANEESHA LAL, 2003). La variable genre devient une variable parmi les autres, signifiante à l'école. ${ }^{10}$

\section{L'adoption du genre dans toutes les disciplines: de nouveaux savoirs potentiellement fondateurs d'une mixité sociale et scolaire moins inégalitaire}

Entre hommes et femmes, il y a du semblable et du dissemblable : « en Philosophie, cela s'appelle une aporie, question ouverte à jamais » note Geneviève FRAISSE (in TOUATI, 2005, p.68). Le concept de genre a le vrai mérite de révéler «l'androgynie psychologique » de chaque personne qui doit construire son identité propre, révéler ses potentialités, développer ses compétences individuelles - qu'elles soient "connotées » féminin ou masculin - en se libérant des injonctions, non-dits ou interdits familiaux et sociétaux, transmis en particulier par l'école. Le domaine de l'éducation est exemplaire du transfert d'apports outre-atlantique et européen : les travaux de Sandra BEM (1974) ${ }^{11}$ contestent les échelles traditionnelles de masculinité et de féminité puisqu'ils débouchent sur des qualificatifs connotés « féminin » ou « masculin » qui sont indépendants - au lieu de complémentaires - et sur "l'androgynie psychologique» de la personne: ils influencent les études ultérieures de Cendrine $\mathrm{MARRO}^{12}$ en classes de 3ème sur l'identité sexuée. Cette pensée subversive révolutionne le regard du maître porté sur l'élève : celuici est perçu désormais comme une «personne en devenir dans sa complétude » au lieu d'un « garçon » ou d'une « fille », équivalent d'un délit de faciès.

L'histoire genrée étudie les relations humaines. Fabrice VIRGILI (2002) préconise de passer « d'une histoire sans les femmes, naguère - déni historique décrit par Michelle Perrot, Françoise THEBAUD - à une histoire du monde sexuée, de nos jours [p. 5]... c'est-àdire à une histoire des relations entre les sexes qui ne soit pas seulement celle de la domination masculine [p. 10]». Une histoire fondée sur un universalisme paritaire, équitable, qui tienne compte des masculinités. Lors des 7èmes Rencontres de l'Histoire à Blois en octobre 2004, Françoise THEBAUD, présidente de Mnémosyne, anime la table ronde « Histoire des femmes et du genre » où l'on expose comment la recherche, appuyée initialement par les féminismes des années 1970 a su se nourrir du militantisme mais pour accéder à l'accréditation universitaire. 
L'approche spatiale (SEAGER) permet de mesurer l'ampleur des inégalités de genre (familles, travail, pouvoir politique, économique) avec des thèmes novateurs et signifiants: la préférence pour l'enfant mâle, le corps, les biens. Les résumés des communications du colloque lyonnais de mars 2004 «Genre, territoire, développement: quels regards géographiques?» questionne la totalité des problématiques de cette discipline jusqu'à l'odonymie qui démontre l'invisibilité symbolique des femmes dans les espaces urbains (MOSQUETE) et innove avec des thèmes historicisés comme le revoilement (SAIDI-SHAHROUZ) dans la sphère publique. L'Harmattan a publié en 2003 une étude pluridisciplinaire qui compare en Sociologie, Histoire et Littérature « Le genre comme catégorie d'analyse » sous la direction de Dominique FOUGEYROLLAS, Christine PLANTE, Michèle RIOT-SARCEY et Claude ZAIDMAN.

Margaret MARUANI (2005) le confirme ${ }^{13}$ « Le genre n'est pas un domaine spécialisé, c'est une grille de lecture de la société. Que l'on s'intéresse à l'école, à l'emploi, à l'immigration, à la famille, à la santé... le genre est un des axes essentiels de la connaissance, un outil indispensable à l'intelligence du monde social ».

Mais l'enjeu des recherches genrées en France, après le tournant que représentent la conscientisation des meilleurs résultats scolaires des filles (DURU-BELLAT, 1990 et BAUDELOT \& ESTABLET 1992) ${ }^{14}$ et l'inadéquation avec les choix filles - garçons en terme de filières d'orientation (Françoise VOUILLOT $1999^{15}$, Catherine MARRY) vise désormais à l'école, lieu d'observation privilégié des effets de domination et de socialisation différenciée des sexes, la (re)fondation de la mixité. D’autant que les travaux de Catherine VIDAL $(2002,2006)$ viennent infirmer les théories scientistes déplorables sur le prétendu "sexe du cerveau» et dénoncer tout "déterminisme biologique» susceptible d'inférioriser les filles - femmes : le traitement différencié garçon - fille à l'école n'a plus aucune justification. Ainsi cette contagion disciplinaire ne peut manquer d'impliquer favorablement l'école, dans la transmission des savoirs (trans-) disciplinaires mais aussi des compétences et des attitudes (cf. le " socle commun »).

\section{L'identité personnelle et professionnelle du maître en question}

Le «connais-toi toi-même» du maitre impose au plan de la connaissance intra personnelle, un regard genré : une pensée réflexive sur la construction de sa propre identité sexuée (par ex. quelle négociation des tâches dans le couple souhaite-t-il/elle ? quel métier et quelle place dans la sphère publique projette-t-il/elle pour son - sa partenaire? son - ses propres enfants?) ...induisant ses propres attentes à l'égard de l'élève - garçon/fille. Et « l'effet Pygmalion » (ROSENTHAL) l'invite à la vigilance au plan professionnel puisque les élèves ont tendance à se conformer aux attentes de leurs enseignant(e)s et à ressembler à leurs représentations genrées et attentes stéréotypées, ceci pour les satisfaire, dans l'espoir de gagner en retour au plan relationnel et affectif. Les stéréotypes constituent un ensemble de représentations inconscientes ou de croyances - et non de connaissances - qui parasitent la réflexion, marquant les préjugés et les comportements car ils induisent des scripts descriptifs pseudo - cognitifs et même prescriptifs. La recherche contemporaine s'applique à faire ressortir comment l'école joue un rôle important dans la transmission des stéréotypes de genre, d'où la nécessaire conscientisation de ses acteurs ${ }^{16}$. Les stéréotypes (LEYENS, J- Ph. 2003 ${ }^{17}$ ) analysés sous l'angle de l'élaboration et de la transmission deviennent des leviers de résistances 
sociétales qu'il faut réduire et modifier: outils a contrario pour les formateurs, enseignants et éducateurs.

\section{Les contenus de la formation à la mixité}

En IUFM, les premiers modules relatifs à la mixité et à l'égalité sont initiés dans la mouvance de la troisième "Convention pour la promotion de l'égalité des chances entre

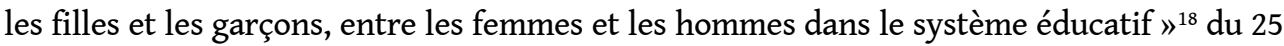
février 2000. D'après l'enquête ciblée de 2005, «seuls 8 IUFM sur 27 dispensent des enseignements sur le genre ${ }^{19}$ » et ce chiffre a progressé à partir de 2002. Selon Michelle ZANCARINI-FOURNEL (2004), en 2003, presque la moitié des IUFM intègrent des enseignements sur la mixité et le genre, selon des modalités variées et face à des publics différents ${ }^{20}$. Cependant la proposition 6 de Nicole BELLOUBET-FRIER, alors rectrice de Toulouse : « Permettre, à tous les PE2 et PLC2 de suivre un module de formation consacré à l'égalité des chances entre filles et garçons dans le système éducatif » n'a pas été suivie de l'effet escompté.

L'étendue des savoirs et des pratiques éducatives et pédagogiques qu'il faudra généraliser constitue, en effet, une véritable re-fondation de la mixité scolaire à visée sociétale égalitaire. L'anomalie vient du fait que les apports transdisciplinaires et des sciences de l'éducation forment un corpus très riche et maîtrisé, véhiculant depuis plus d'une quinzaine d'années de très nombreux outils et de nouvelles pratiques - souvent mis en ligne, réalisés en appui sur les Conventions interministérielles Égalité filles - garçons mais qui ne sont connus que d'un petit nombre de praticiens de l'éducation ou de la formation, travaillant souvent en réseaux ${ }^{21}$ (MOSCONI, 1998).

En amont de cette (re-) fondation, le «discours de l'enseignant(e)» est au cœur du rééquilibrage : il doit intégrer et valoriser du «féminin »... L'adoption au Canada de l'expression «droits de la personne » au lieu de «droits de l'homme» est exemplaire. Face à la symbolique du langage, au primat du masculin, des réponses fortes ont déjà été données, telle la Circulaire Lionel Jospin sur "la féminisation des noms de métier, fonction, grade ou titre» de 1998. Mais c'est un chantier infini qui débute car la langue française consacre l'invisibilité des filles/femmes par l'utilisation quasi exclusive du masculin dit "générique, universaliste, républicain »; l'accord de proximité - aboli par Vaugelas en 1647 - n'en constituant qu'un aspect (Claudie BAUDINO, 2005) ${ }^{22}$. La convocation du « double sujet » par le (la) professeur(e) est une pierre à l'édifice : « ils et elles $» . .$.

Si la conscientisation du genre, c'est-à-dire d'une construction culturelle des rôles de sexe et du masculin - féminin dès la petite enfance (ACHERAR, 2003) est à la base de toute formation, le maître doit questionner les normes collectives de la socialisation différenciée à l'école, l'inclusion normative « conditionnant » dès l'enfance implicitement ou explicitement par les choix d'activités, de jeux, d'interactions (DAFFLON NOVELLE, 2006). Au collège la dynamique de classe passe par un décryptage du langage et des interrelations élèves / enseignant(e), dont les injonctions ou interdits voire les non-dits (dé) valorisants «travaillent» les identités sexuées et stratégies scolaires. Même «l'interpellation de l'élève » n'est pas neutre et doit s'ajuster aux cultures et valeurs familiales ; l'élève doit être sollicité(e) comme une personne et non traité(e) « en fille » ou « en garçon ». L'espace vocal doit être réajusté à l'encontre de la loi dite des 2/3 (Marie DURU-BELLAT, 1990) : il faut plus solliciter les filles, connaitre leurs prénoms, sinon elles 
restent plus discrètes. La classe mixte ne doit pas cantonner les filles à reformuler répéter - résumer soit à "l'auxiliariat pédagogique " (Claudine BLANCHARD-LAVILLE, 1997) ${ }^{23}$ mais les appeler à réfléchir - innover - inventer. Le maître doit aussi savoir repérer « les pratiques langagières » des adolescent(e)s et la pression normative des pairs dans la classe (Florence MAILLOCHON, 2003) ${ }^{24}$.

Chantier majeur de la formation que d'explorer pour les dé - reconstruire les représentations collectives débouchant sur un traitement différencié en matière d'éducation, d'évaluation - orientation diagnostique, et des savoirs et choix de métiers sexués (GAUTHIEZ-RIEUCAU, 2007).

La sémiologie, science nourrie par la pensée de Roland BARTHES, traque les signes omniprésents à travers la publicité, la signalétique, les arts visuels, etc. et l'enseignant doit savoir aussi décortiquer toutes formes de «langages " vestimentaire, alimentaire, musical... destinés aux jeunes. Pression médiatique lourde contre laquelle l'école a du mal à lutter, dans le contexte de la relance commerciale des «cultures binaires »: «culture fille» et "garçon" sont hypervisibles dans les agendas de rentrée. Cette binarité va exactement à l'encontre de la mixité citoyenne, paritaire préconisée à l'école par les textes officiels à la lumière des acquis sur le genre.

À l'école, les savoirs «savants ou innovants » (MOSCONI, 1999) - les disciplines ellesmêmes étant connotées (MORIN, 1997) - comme les orientations induites et les choix de filières - métiers, sont sexués et doivent être revisités... La chasse aux stéréotypes de genre, insuffisante dans les programmes, implique une formation à un choix lucide des manuels. À tous les niveaux de l'éducation, les manuels constituent ainsi des supports inépuisables de la transmission des stéréotypes de genre, non seulement à travers les textes mais aussi l'iconographie proposée... La lutte contre le sexisme dans les manuels scolaires, initiée par Françoise GIROUD en 1975 pour la petite enfance, s'est concrétisée et démultipliée à partir du ministère de Yvette ROUDY en 1981 à tous les niveaux de l'enseignement. Le travail d'analyse critique des manuels se poursuit dans l'esprit du Rapport parlementaire RIGNAULT et RICHERT de 1997. La relative mais persistante « invisibilité des femmes» demeure un obstacle majeur - directement palpable et quantifiable dans les manuels d'histoire - comme le pointe le rapport d'Annette WIEVIORKA au Conseil économique et social en 2004 ; les stéréotypes sont dénoncés dans toutes les disciplines, à titre d'exemples, en anglais, par Anne GRIFFATON (1999), en sciences économiques et sociales par Jane MEJIAS $(2005,2006)$ ou en sciences et technologies de la gestion par Christine FONTANINI (2007); albums et manuels sont désormais ciblés au plan international. ${ }^{25}$

Comme l'école devient le lieu obligé d'une interdisciplinarité féconde, en particulier entre psychologie sociale et sciences de l'éducation - les travaux sur la valence différentielle de la catégorie de sexe en terme de perception de soi de Marie-Claude HURTIG et MarieFrance PICHEVIN (colloque européen d'Aix 1986) ${ }^{26}$ sont corrélatives des études de relations intergroupes dans le cadre de la mixité à l'école (Fabio LORENZI-CIOLDI $1988{ }^{27}$ et Annick DURAND-DELVIGNE 1990 ${ }^{28}$ qui font ressortir le risque de renforcement des stéréotypes de genre en classe mixte - il y a nécessité à (re-)fonder de nouvelles pratiques égalitaires en classe mixte et en formation. Nicole MOSCONI publie «La mixité dans l'enseignement secondaire: un faux-semblant?» dès 1989 et le collectif «Égalité des 
sexes en éducation et formation "suscité en découlera en 1998. Pour autant, Mireille BAURENS (2006) continue à dénoncer "une mixité unisexe, à savoir une mixité apparente, qui dysfonctionne" - eu égard, entre autres, à la pédagogie de l'encouragement différencié, à la topographie des classes et activités, aux regroupements des élèves en cours et dans la cour, aux interactions en classe, à l'évaluation et à l'orientation - et « une mixité que les enseignant(e)s n'interrogent guère ».

$\mathrm{Au}$ sein du corpus, nombreux sont les apports des sciences de l'éducation ${ }^{29}$ qui mériteraient d'être mis à l'épreuve de la classe systématiquement par les stagiaires d'IUFM et/ou analysés dans des dossiers ou mémoires professionnels: l'auxiliariat pédagogique; le double standard et le curriculum caché (FORQUIN) ; l'effet Pygmalion (ROSENTHAL); l'effet "menace du stéréotype » (Spencer, 1999) 30 et "l'effet solo » (SEKAQUAPTEWA et THOMSON, 2002) ${ }^{31}$; les notions de "groupe agrégat " pour les filles et de " groupe collection " pour les garçons; la loi dite initialement « des $2 / 3$ » ou plutôt l'espace vocal et pédagogique attribué dans les interrelations à chaque sexe; enfin les situations d'apprentissage mixtes/non mixtes (Verena AEBISCHER, 1998) ${ }^{32}$; l'évaluation diagnostique relative aux orientations sexuées, etc. autant de remise en question des pratiques impliquant l'identité professionnelle.

En formation continue aussi, l'on peut interroger des grilles d'observation de classe incluant tous ces apports et questionner les effets groupes mixtes ou non mixtes qui imprègnent le quotidien ...

L'on peut recenser des pratiques contribuant à (re)fonder une mixité citoyenne de type égalitaire :

- traquer le « double - standard » sur l'éduquer, l'évaluer et l'orienter

- favoriser des groupes de travail unisexes et organiser une alternance de l'expression et des pratiques intergroupes d'intérêt commun,

- développer des groupes mixtes et des pratiques intragroupes mixtes de situations de coopération entre les filles et garçons (et non pas de type de compétition),

- développer les situations de renforcement de la cohésion du groupe-classe mixte face à des enjeux externalisés,

- faciliter l'émergence de la parole féminine et de l'altérité-égalité par l'alternance de l'expression individuelle,

- éduquer à la critique des stéréotypes dans le vécu, les manuels et matériel pédagogique et conscientiser les rôles de sexe,

- distribuer à parité les tâches et missions de responsabilité dans la classe et l'Institution.

Les contenus de formation évoluent à l'aune des riches travaux interdisciplinaires que continuent à mener des chercheurs qui ont contribué à vulgariser le concept de genre sur le terrain - tels Michelle ZANCARINI-FOURNEL avec le Fonds Aspasie de l'IUFM de Lyon, Nicole MOSCONI, Françoise VOUILLOT du CNAM-INETOP - et d'autres, dont les disciplines d'origine sont multiples, qui diversifient les apports et propositions de nouvelles pratiques de la mixité: Christine MORIN, Christine FONTANINI ou encore Bénédicte GENDRON (2007, et dans ce numéro) : l'école, « reproductrice des stéréotypes sociaux de sexe » ne développe pas les mêmes compétences émotionnelles chez les filles (empathie, 
autorégulation, compétences " plus scolaires ») que chez les garçons (assertivité d'où sous - réalisation dans le travail scolaire et " plus de compétitivité professionnelle») «ce qui apporte des éléments d'explications aux différences observées en matière de performances scolaires et d'orientation professionnelle», autrement dit, là aussi la formation à la mixité doit réduire l'asymétrie.

Le travail en réseaux, interuniversitaire et inter académique, se poursuit de plus en plus concrètement et au niveau européen.

Armelle LEBRAS-CHOPARD (2004, chap.17, p 349-375) dresse un bilan actif de «la politique d'égalité dans l'Enseignement supérieur au ministère de l'Éducation nationale ${ }^{33}$ ", basé en particulier sur les grandes enquêtes nationales mises en ligne et Catherine MARRY (2004, chap.16, p 343) évalue positivement les politiques de diversification d'offre scolaire - notamment l'ouverture des classes préparatoires scientifiques ou des sections d'IUT dans des villes moyennes et des internats pour les filles - et nuance les effets des Conventions sur la diversification de l'orientation des filles: "C'est plutôt la relative inefficacité des politiques scolaires qui visent à infléchir les orientations des filles (celles des garçons sont moins critiquées) qui est la plus manifeste. La division sexuée des savoirs et des métiers semble résister autant que les inégalités sociales. ${ }^{34}$ »

Une autre étape apparaît nécessaire si l'on veut en finir avec cette opposition entre un discours politique explicite fondé sur une société égalitaire... et une réalité scolaire et sociale de terrain implicite inégalitaire, dans un contexte où la mondialisation complexifie et enrichit les jeux de l'intra et de l'inter culturalité... étape qui suppose de mettre en œuvre les Conventions interministérielles Égalité Filles - Garçons - lesquelles impliquent en outre la dimension capitale de l'éducation au respect mutuel de l'autre sexe et la prévention des violences sexistes et sexuelles ${ }^{35}$ - soit de généraliser en les rendant obligatoires les formations sur la mixité auprès des enseignants, en formation initiale et continue et auprès de tous les acteurs de l'éducation, dont les chefs d'établissement, les conseillers d'éducation et d'orientation, dans une démarche politique intégrée pour contrer les inégalités de genre.

Ecoutons les voix de Françoise THEBAUD et de Michelle ZANCARINI-FOURNEL (2003, p.18): «L'enjeu des débats sur la coéducation pour les pédagogues du XIXe est comparable aux interrogations qui naissent dans les sociétés du XXIe sur la coexistence des deux sexes dans un espace mixte. S'intéresser à l'histoire de la mixité scolaire, c'est interroger l'historicité des institutions scolaires et aussi celle du rapport social entre les sexes à la faveur des acquis sur le genre. » 


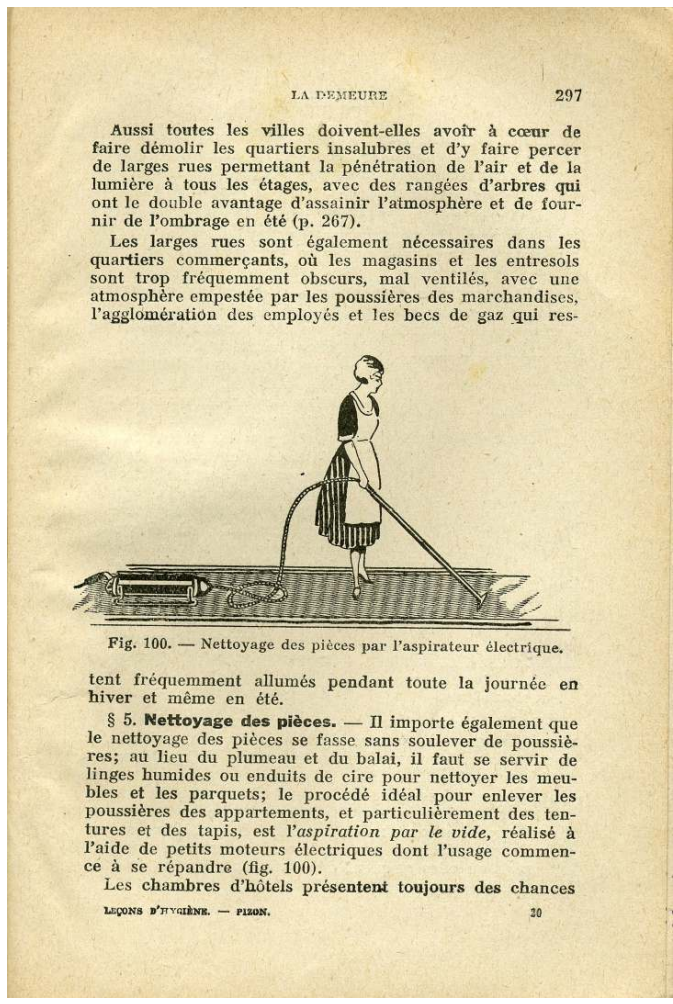

Stéréotype de genre dans un manuel d'hygiène. Image tirée de Leçons d'hygiène à l'usage de la classe de $3^{\mathrm{e}}$ garçons et filles / Antoine Pizon. Paris : Doin \& Cie, 1930. 310 p. : ill. ; 19 cm.- p. 20. Source : CEDRHE

\section{BIBLIOGRAPHIE}

ACHERAR, L. (2003). Filles et Garçons à l'école maternelle. Rapport. www.droits-femmes-lr.fr/pdf/ $\underline{\text { maternelle }}$

Actes du colloque Les violences sexistes à l'école, janvier 2003. Textes réunis par Dominique GAUTHIEZ-RIEUCAU, IUFM de Montpellier, mars 2004.

Actes du colloque Quelles mixités construire à l'école ? IUFM de Montpellier, 17 novembre 2004. p. 49. Textes réunis par Dominique GAUTHIEZ-RIEUCAU.

B.O. du Ministère de l'Education nationale et de la Recherche, Hors Série, ${ }^{\circ} 10,02$ novembre 2000, Préambule

BARD, Ch., BAUDELOT, Ch. \& MOSSUZ-LAVAU, J. Dirs (2004). Quand les femmes s'en mêlent. Genre et pouvoir. Paris : La Martinière.

BAURENS, M. (2006). Langues-cultures et genre : failles et défis pour la mixitéf-g à l'école, Le genre en éducation I, IUFM Martinique-IUFM Grenoble.

BELLOUBET-FRIER, N. (2001). Trente Propositions pour lutter contre la violence sexuelle dans les établissements scolaires. Rapport. Toulouse : Académie de Toulouse. 
BUTLER, J. (1990). Gender trouble, Feminism and the Subversion of Identity. New York : Routledge. Traduction de Cynthia Kraus, Paris : La Découverte, 2005.

Cahiers de l'INAS. (1965). Rennes : CRDP.

CASTELAIN-MEUNIER, Ch. (1988). Les hommes aujourd'hui, virilité et identité. Paris : Acropole.

DAFFLON Novelle, A. (dir.) (2006). Filles-Garçons, Socialisation différenciée ? Grenoble : PUG.

DE GOUGES, O. Déclaration des droits de la femme et de la citoyenne, septembre 1791.

DELPHY, Ch. (1998). L'ennemi principal. Rééd. 2009, Paris : Syllepses.

DEMEULENAERE-DOUYERE, C. (2003). Un précurseur de la mixité : Paul Robin et la coéducation des sexes, CLIO Histoire, Femmes et Sociétés, n¹8, pp. 132.

FERRAND, M. (2004). Féminin Masculin. Paris : La Découverte, coll. Repères. p. 61.

FONTANINI, Ch. \& PANISSAL, N. (2007). Les nouveaux manuels STG ont-ils repensé les normes et valeurs de notre société concernant le genre ? 3ème Journée Pierre GUIBBERT, IUFM (Actes à paraître).

FOUGEYROLLAS, D. PLANTE, Ch. RIOT-SARCEY, M. \& ZAIDMAN, C. (dir.). (2003). Le genre comme catégorie d'analyse. Paris : L'Harmattan.

GAUTHIEZ-RIEUCAU, D. (2006). L'institutionnalisation du genre. L'exemple de Montpellier, en partenariat avec le Rectorat, les Universités et la vie associative, Genre en Éducation I, L'éducation des filles et des garçons à l'école, IUFM Grenoble \& Martinique.

GAUTHIEZ-RIEUCAU, D. (2007). Les représentations des filles et des garçons à l'école, Actes du séminaire transdisciplinaire sur le genre UPV-Montpellier3. Montpellier : PULM. (à paraître).

GENDRON, B. (2007). Éducation sexuée et capital émotionnel : quelles différences filles - garçons ? Cahiers du Cerfee, $\mathrm{n}^{\circ} 23$, Montpellier III.

GIFFATON, A. (1999). Images des femmes dans les manuels d'anglais, Les cahiers pédagogiques, $\mathrm{n}^{\circ}$ 372.

HURTIG, M.-C. KAIL, M. \& ROUCH, H. (1991). Sexe et genre. De la hiérarchie entre les sexes. Paris : CNRS.

LELIEVRE, F. \& LELIEVRE, C. (1991). Histoire de la scolarisation des filles. Paris : Nathan. MARGUERITTE, V. (1927). Ton corps est à toi. Paris : Flammarion.

MARRY, C. (2004). Les femmes ingénieurs, une révolution respectueuse. Paris : Belin. pp 46-63

MARUANI, M. dir. (2005). Femmes, genre et sociétés l'État des savoirs, Paris : La Découverte. 480 p. MATHIEU, N-C. (1991). L'anatomie politique. Catégorisations et idéologies du sexe. Paris : Côté femmes. MEJIAS, J. (2005). « Genre et manuels de SES », Idées, n¹39, mars.

MEJIAS, J. (2005). La question du genre en sociologie. Paris : Bréal, coll. Thèmes et Débats.

MEJIAS, J. (2006). L'école à l'épreuve de l'actualité. Paris : ESF.

MORIN, Ch. (1997). Mixité et performances scolaires, Cahiers internationaux de psychologie sociale. MOSCONII, N. (1989). La mixité dans l'enseignement secondaire, un faux-semblant? Paris : PUF.

MOSCONI, N. (Coll.) (1998). Éducation des sexes en éducation et formation. Paris : PUF.

RAYNAL, M. (Coord.) (2004). Les filles et les garçons sont-ils éduqués ensemble ? VEI Diversité, Scérén, $\mathrm{n}^{\circ} 138$. 
SCOTT, J. (1986). Gender. A useful category of historical analysis. Trad. Française de RIOT-SARCEY, M. 1988.

SEAGER, J. (2008) The State of Women in the World Atlas (4th ed). Atlas des femmes dans le monde. Paris : Autrement.

THEBAUD, F. \& ZANCARINI-FOURNEL, M. (dirs.), (2003). « Coéducation et mixités », CLIO Histoire, Femmes et Sociétés, n¹8, pp. 11-19.

THERY, I. (1993). Le démariage. Paris : Jacob.

THERY, I. (2001). La mixité, figure nouvelle de l'égalité, in KNIBIELHER, Y. (dir.), Maternité, affaire privée, affaire publique. Paris : Bayard.

TORSAT, D. (1999). Les politiques d'égalité à l'école entre les filles et les garçons, 1984-89, in Françoise VOUILLOT (dir.), Une égalité à construire, Paris : CNDP, coll. Autrement.

TOUATI, A. (dir.) (2005). Actes du Colloque Femmes et Hommes, l'invention des possibles, 2005. Cultures en mouvement.

VIDAL, C. (Dir) (2002). Le cerveau, le sexe et l'idéologie dans les neurosciences, CNAM, 2002, vol 31, n44, pp 495-505.

VIDAL, C. (2006). Féminin Masculin, Mythes et idéologies. Paris : Belin, coll. Regards

VIRGILI, F. (2002). L'histoire des femmes et l'histoire des genres aujourd'hui, Vingtième siècle, $\mathrm{n}^{\circ}$ spécial 75.

ZAIDMAN, C. \& BAUDOUX, C. (1992). Égalité entre les sexes, Mixité et Démocratie. Paris : L'Harmattan.

ZAIDMAN, C. (1996). La mixité à l'école primaire. Paris : L'Harmattan.

\section{NOTES}

1. cf. les \& 1.2 et 2.1

2. «Il faut sauver les garçons! », Le monde de l'éducation, n³10, janvier 2003.

3. Loi HABY de 1975 et Décrets d'application en 1976 qui généralisent l'organisation des classes mixtes à tous les degrés d'enseignement.

4. Rapports de sexe, Rapports de genre Entre domination et émancipation, Revue VEI Enjeux, $\mathrm{n}$ - 128, mars 2002, $254 \mathrm{p}$.

5. Article de Christiane DEMEULENAERE-DOUYERE, « Un précurseur de la mixité : Paul Robin et la coéducation des sexes"

6. In Cahiers de l'INAS, Rennes, CRDP, 1965.

7. Olympe de Gouges, Déclaration des droits de la femme et de la citoyenne, septembre 1791.

8. L'égalité, une utopie ? Cahiers du genre n³3, 2002

9. La distinction entre sexe et genre, une histoire entre biologie et culture, Cahiers du genre $n^{\circ} 34$, 2003

10. Ibid, Sexe, genre et historiographie féministe contemporaine : l'exemple de l'Inde coloniale, Cahiers du genre $\mathrm{n}^{\circ} 34,2003$, p149-167

11. BEM, S. (1974) The measurement of psychological androgyny, Journal of Consulting and Clinical Psychology, p155-162

12. Construction de l'identité chez les filles et les garçons, les femmes et les hommes dans notre société, CNAM, 2002, vol 31, n², pp 545-563

13. L'ouvrage collectif qu'elle dirige rassemble 58 auteur-e-s appartenant aux différentes disciplines des sciences humaines et sociales: sociologues, démographes, historien(ne)s, 
économistes, statisticien(ne)s, philosophes, anthropologues, juristes, politologues, épidémiologistes, ergonomes. Cf. p12

14. DURU-BELLAT, M. L'école des filles. Quelles formations pour quels rôles sociaux? Paris: L'Harmattan; puis BAUDELOT, C. § ESTABLET, R. Allez les filles! Paris : Seuil, réédition coll Points actuels

15. VOUILLOT, F. (Dir.), Filles et garçons à l'école: une égalité à construire, Paris: CNDP, coll. Autrement

16. Jeanne FINE, Equality of the sexes: Research and Training in the IUFM et Jean-Paul FILIOD, Des hommes parmi les femmes : être et devenir professeurs des écoles, cités en intro et conclusion $\mathrm{p} 92$ dans «École et mixités », Annik HOUEL et Michelle ZANCARINI-FOURNEL, Cahiers Masculin/Féminin, PUL, 2001

17. Les stéréotypes in Sciences humaines, $n^{\circ} 139$

18. La 1ère Convention interministérielle Egalité F-G et F-H date de 1984 (Y. ROUDY/J-P. CHEVENEMENT), la 2e de 1989 (M. ANDREE/R. CHAPUIS); la 3e signée le 25 février 2000 (C. ALLEGRE/N. PERY) est élargie à d'autres ministères et prorogée 2004-06 (LANG/PERY); la 4e couvre 2006-2011 (de ROBIEN/VAUTRIN)

19. Revue Passerelles, ${ }^{\circ} 6$, avril $2005, \mathrm{p} 3$.

20. Actes du colloque Quelles mixités construire à l'école ? IUFM de Montpellier, 17 novembre 2004. p. 49. Textes réunis par Dominique Gauthiez-Rieucau. Michelle ZANCARINI-FOURNEL est alors Chargée de mission nationale Egalité pour les IUFM : cf. Formations de Formateurs et Groupes d'analyse de pratiques (pp78-82)

21. L'ouvrage, fruit de la 3ème Biennale de l'Éducation et de la Formation, « s'adresse à tous ceux, administrateurs, enseignants, formateurs, éducateurs que les questions de l'éducation des filles et des garçons, de la formation des hommes et des femmes interrogent et préoccupent et qui cherchent, sur ce thème, informations, réflexions et suggestions ".

22. Claudie BAUDINO, Politique de la langue et différence sexuelle, la politisation du genre des noms de métier, 2001, Paris, L'Harmattan, 364 p. ouvrage présenté par Mireille BAURENS dans Histoire Clio, Femmes et Sociétés, $\mathrm{n}^{\circ} 22,2005$

23. Variations sur une leçon de mathématiques. Savoir et formation, Paris : L'Harmattan

24. Le jeu de l'amour et de l'amitié au lycée : mélange de genres, Travail, Genre et Sociétés, avril $2003, n^{\circ} 9$

25. Par exemple Analyser les représentations du masculin et du féminin dans les manuels scolaires (Collectif du XXVe Congrès international de la population : chercheurs européens et africains dont Carole BRUGEILLES, Sylvie CROMER, LOCOH), CEPED, 2005

26. Actes du colloque national Femmes, féminisme et recherches de 1984 HURTIG, M.C. and PICHEVIN, M.F. (1986)

27. LORENZI-CIOLDI, F. Individus dominants et groupes dominés images masculines et féminines, PU Grenoble, juin 1988 et Les représentations des groupes dominants et dominés: collections et agrégats Grenoble, PU Grenoble, 2002, $360 \mathrm{p}$

28. DURAND-DELVIGNE, A. Jeu du soi et du genre: les effets structurels de la co-éducation, Cahiers pédagogiques, $\mathrm{n}^{\circ} 280,1990$

29. Marie RAYNAL (Coord.), VEI Ville, Ecole, Intégration - Diversité, ${ }^{\circ}{ }^{\circ 138,} 2004$; cf. en particulier Christine Morin, «Contexte scolaire et mixité » p 73 ; et parmi les chercheurs évoqués, sans que la liste soit exhaustive mais indicative, dans l'ordre d'apparition dans le texte des théories et propositions de nouvelles pratiques de classe: Claudine BLANCHARD-LAVILLE, FORQUIN, ROSENTHAL; SPENCER ; SEKAQUAPTEWA et THOMSON Ibid. p. 34 ; Fabio LORENZI-CIOLDI et Annick DURAND-DELVIGNE ; Mireille BAURENS ; VERENA AEBISCHER ; en matière d'orientations cf. Françoise VOUILLOT

30. Under suspicion of inability : stereotype threat and women's math performance, Journal of Experimental Social Psychology, $\mathrm{n}^{\circ} 35, \mathrm{p}$ 4-28 
31. The differential effects of solo status, Personality and Social Psychology Bulletin, $\mathrm{n}^{\circ} 28, \mathrm{p}$ 694-707 ; et dans VEI ${ }^{\circ} 138, \mathrm{p} 34$

32. Styles, communautés de pensée et performances scolaires, p 110-123 et en particulier p 117 in Nicole MOSCONI (Dirs), 1998, Egalité des sexes en éducation et formation, PUF

33. Armelle LEBRAS-CHOPARD in Christine BARD, Christian BAUDELOT, Janine MOSSUZ-LAVAU (Dirs), Quand les femmes s'en mêlent. Genre et pouvoir, Ed. La Martinière, 2004, Chap. 17, pp. 349-375 34. Catherine MARRY, ibid. in Christine BARD, Christian BAUDELOT, Janine MOSSUZ-LAVAU (Dirs.), Quand les femmes s'en mêlent. Genre et pouvoir, Ed. La Martinière, 2004, Chap. 16, p. 343

35. Actes du colloque Les violences sexistes à l'école, tenu en janvier 2003, Textes réunis par Dominique GAUTHIEZ-RIEUCAU, IUFM de Montpellier, mars 2004

\section{RÉSUMÉS}

La mixité scolaire est inaccomplie car elle a été installée de manière pragmatique et doit être (re)fondée dans une perspective d'égalité - altérité. La nécessaire conscientisation du genre et de la socialisation différenciée garçons - filles implique l'identité personnelle et professionnelle des enseignants dans le cadre d'une formation transdisciplinaire à l'éduquer - évaluer - enseigner orienter qui vise à réduire l'asymétrie.

Coeducation has only been partially implemented, because the concern has been purely practical ; it must be (re)established to take into account equality and difference. The necessary gender and male-female socialisation awareness implicates personal and professional engagement of teachers, via a transdisciplinary training course to educate, evaluate, teach and guide, in order to reduce the unbalance.

\section{INDEX}

Mots-clés : androgynie psychologique, compétence individuelle, curriculum caché, double standard, effet groupe mixte/non mixte, effet Pygmalion, genre, stéréotype

Keywords : double standard, effect of co-ed/single-sex groups, gender, hidden agenda, individual ability, psychological androgyny, Pygmalion effect, stereotype

\section{AUTEUR}

\section{DOMINIQUE GAUTHIEZ-RIEUCAU}

Professeure certifiée en histoire - géographie, DEA de civilisation européenne, travaux de recherche en Histoire contemporaine (Archives Jean Rous) et transdisciplinaires sur le genre, Personnel de direction Éducation nationale 